\title{
Superior Semicircular Canal Dehiscence Syndrome without Vestibular Symptoms
}

\author{
Emidio Oliveira Teixeira ${ }^{1}$ Marconi Teixeira Fonseca ${ }^{1}$ \\ ${ }^{1}$ Department of Otorhinolaryngology and Head and Neck Surgery, \\ Socor Hospital, Belo Horizonte/MG, Brazil \\ Int Arch Otorhinolaryngol 2014;18:210-212.
}

Address for correspondence Emidio Oliveira Teixeira, MD, Rua Garret, 581, apto 301, Bairro Nova Granada, Belo Horizonte/MG, CEP 30431 355, Brazil (e-mail: emidio_teixeira@hotmail.com).

\author{
Abstract \\ Keywords \\ - semicircular canals \\ - ear \\ - inner \\ - tinnitus
}

Introduction Superior semicircular canal dehiscence syndrome is mainly characterized by vestibular symptoms induced by intense sound stimuli or pressure changes, which occur because of dehiscence of the bony layer covering the superior semicircular canal.

Resumed Report Here, we report a case of the syndrome with pulsatile tinnitus and ear fullness, in the absence of vestibular symptoms.

Discussion Signs and symptoms of the syndrome are rarely obvious, leading to the requirement for a minimum workup to rule out or make diagnosis more probable and thus avoid misconduct.

\section{Introduction}

Superior semicircular canal dehiscence syndrome (SSCDS) was described in 1998 by Minor et al. ${ }^{1}$ Its prevalence in the general population is estimated at $0.7 \%$ for total dehiscence and $1.3 \%$ for the presence of a thin layer covering the bone channel $(\leq 0.1 \mathrm{~mm}){ }^{2}$

There is no evidence of significant sex predominance, and the median age of symptom onset is around 40 to 41 years. ${ }^{3}$ The anatomical change responsible for SSCDS is still of unknown etiology, although it could occur as a result of abnormalities during development of the bony layer that covers the semicircular canal (at up to 3 years old) and the occurrence of a secondary event (head injury or sudden increase in intracranial pressure) leading to rupture of an abnormally thin bony layer and complete dehiscence. ${ }^{2} \mathrm{Al}-$ though vestibular manifestations are more frequent, some patients may only have auditory symptoms. ${ }^{4}$

\section{Case Report}

The 65-year-old woman was a housewife born in Belo Horizonte-MG and resided in this city. She had a history of 11 years of recurrent left ear fullness with autophonia and ipsilateral pulsatile tinnitus; as her symptoms were especially nocturnal, they led to frequent wakening. She reported a recent increase in the seizure frequency of ear fullness and worsening of her symptoms resulting in anxiety, and she denied a relationship with change in body position or exertion. She used clonazepam irregularly, which caused partial improvement of her tinnitus. She denied vertigo, hearing loss, ear pain, and the occurrence of major head trauma.

The patient had hypertension and used amlodipine, atenolol, and chlorthalidone. She also suffered from a depressive disorder, for which she was not taking any medication. She showed no abnormalities upon an ear, nose, and throat physical examination. Her laboratory tests indicated a normal metabolic profile, and duplex scanning of the carotid artery was unchanged.

An audiogram descending from $500 \mathrm{~Hz}$ showed symmetrical paths and a type A tympanometric curve ( - Fig. 1). Upon a stapedial reflex test with a testing frequency of $110 \mathrm{~Hz}$ and an intensity of $110 \mathrm{Db}$ in the right ear (left ear probe), the patient had vertigo and nystagmus (Tullio phenomenon). Magnetic resonance imaging (MRI) was requested and subsequent computed tomography (CT) of the temporal bones with thin slices and sagittal reconstruction was performed, which showed signs suggestive of SSCDS on the left, compatible with the third window (-Figs. 2-4).

The patient received guidance and was reassured about the nature of the syndrome. A conservative approach with regular clinical monitoring was chosen as a result of the received

October 6, 2011

accepted

November 16, 2011
DOI http://dx.doi.org/

$10.1055 / \mathrm{s}-0033-1351670$. ISSN 1809-9777.
Copyright $(2014$ by Thieme Publicações License terms Ltda, Rio de Janeiro, Brazil 


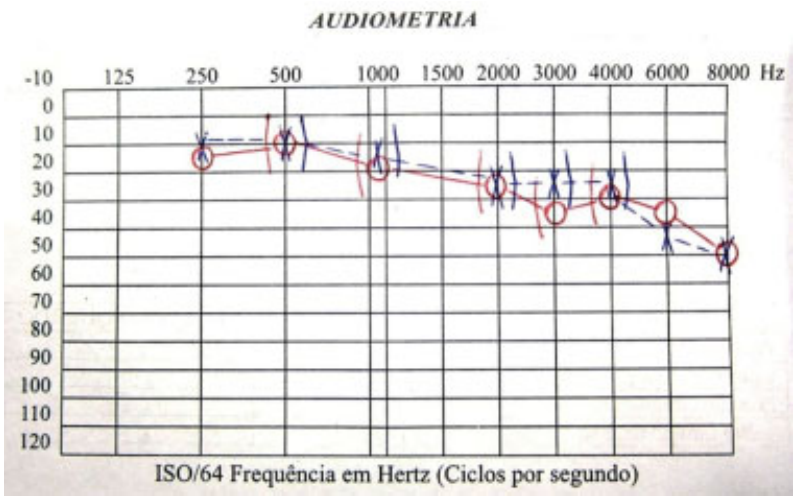

Fig. 1 Symmetric audiometric tracings, descending from $500 \mathrm{~Hz}$.

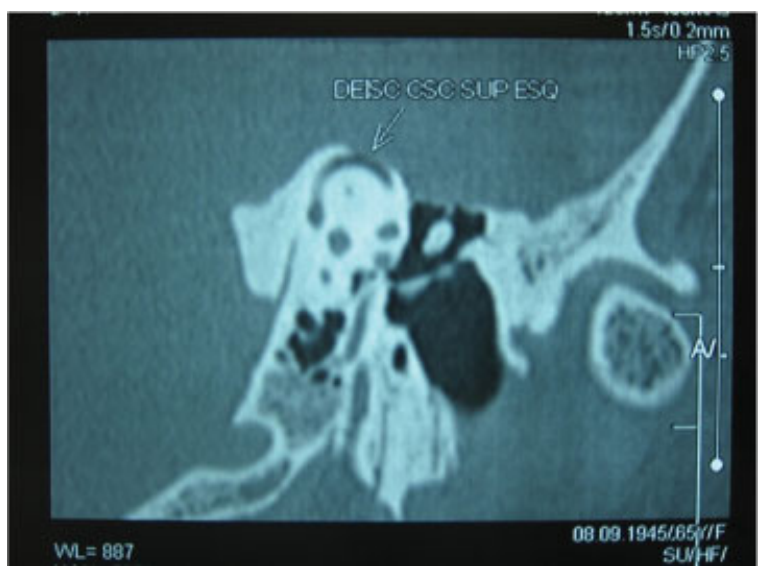

Fig. 2 Left superior semicircular canal dehiscence as seen on sagittal temporal bone computed tomography.

absence of disabling symptoms and classification of the presentation as light.

\section{Discussion}

Most patients with SSCDS seek medical care for symptoms and vestibular nystagmus triggered by noise or pressure change (e.g., blowing nose, coughing, lifting heavy objects, and compression of the tragus). The patient may refer to hearing ipsilateral eye movements, their heartbeat, or popping of the joints during movement. A minority of patients seek care for hearing loss only, without vestibular symptoms. ${ }^{1,3,5,6}$

In a review and analysis of clinical data, Minor observed that 65 patients had confirmation of superior semicircular canal dehiscence upon high-resolution CT of the temporal bone and the presence of at least one signal indicating physiologic testing for superior semicircular canal dehiscence. ${ }^{4}$ Vestibular manifestations were present in 60 (92.3\%) patients, whereas only auditory manifestations without vestibular symptoms or signs were noted in $5(7.7 \%)$ patients. ${ }^{4}$

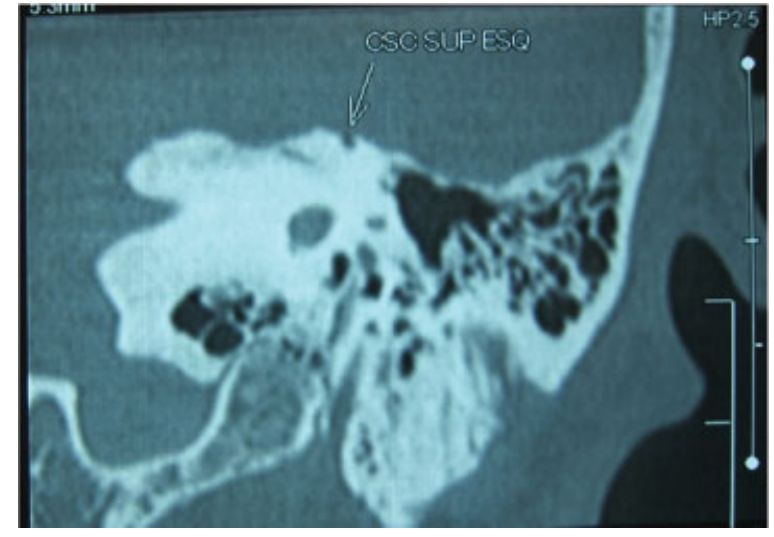

Fig. 3 Left superior semicircular canal dehiscence as seen on coronal temporal bone computed tomography.

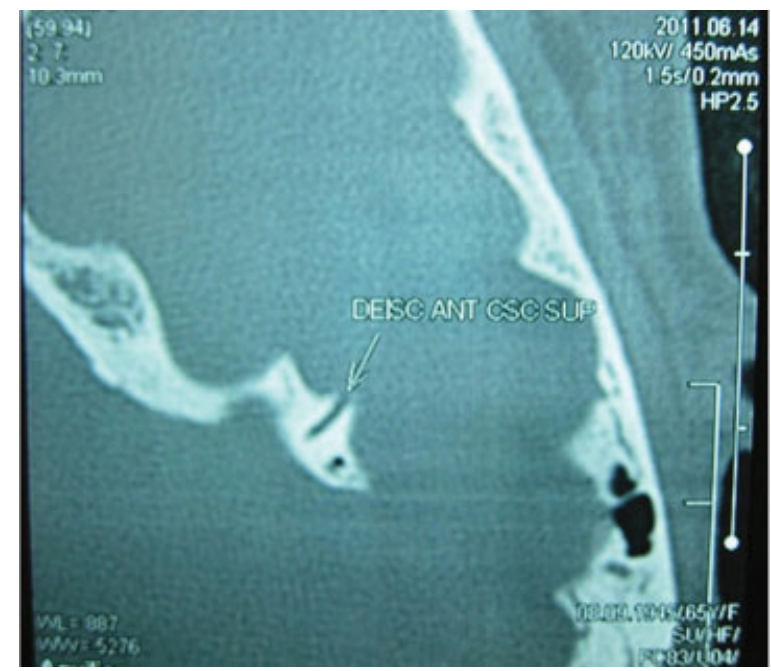

Fig. 4 Left superior semicircular canal dehiscence as seen on axial temporal bone computed tomography.

In the current case, the patient reported pulsatile tinnitus and fullness in the ear without vertigo symptoms. Nam et al described a case of SDCSS where the only complaints were tinnitus and autophonia in the right ear induced by repeated rotations of the head in the plane of the right semicircular canal with an angular velocity greater than 600 degrees per second. ${ }^{7}$

Hearing alterations occur as the result of the introduction of a third window in the inner ear, which may reduce the cochlear input impedance, create "shunts" of air escaping sound conduction in the cochlea, and result in conductive hearing loss and increased bone conduction thresholds, increasing the difference in impedance between the vestibule and the round window. ${ }^{8}$ Although more studies are needed to determine why some patients with SSCDS have only a few purely auditory symptoms but others manifest both vestibular and auditory symptoms, patency of the cochlear aqueduct and the relative complacency of the round window membrane are factors that may contribute to the existence of this difference. ${ }^{4}$ 
Hearing loss that is conductive at low frequencies is the most common finding of SSCDS. There is usually an air-bone gap of 5 to $10 \mathrm{~dB}$ in two or more frequencies, although a larger gap may exist. Patients generally have a preserved acoustic reflex upon tympanometry and respond to the vestibular evoked myogenic potential test. Sensorineural hearing loss and mild to moderate conductive hyperacusis (decreased bone conduction threshold within normal air conduction) are also observed. ${ }^{1,3,9}$ When dehiscence is greater than $3 \mathrm{~mm}$, an air-bone gap is presented consistently at low frequencies, and the higher the dehiscence, the higher the air-bone gap upon audiometry. ${ }^{10}$

Diagnosis is confirmed by high-resolution temporal bone CT scanning. The positive predictive value increased to a dehiscence of $50 \%$ using CT with $1.0-\mathrm{mm}$ slices and images in the axial and coronal planes and to $93 \%$ using thin slices $(0.5 \mathrm{~mm})$ and reconstruction in the sagittal plane. ${ }^{11}$ However, the prevalence of superior canal dehiscence present in research on thin sections of temporal bone reconstruction in the sagittal plane is much larger than that predicted by pathologic studies, so the risk of overdiagnosis is present. ${ }^{12}$ MRI was found to have a sensitivity of $96 \%$ and a specificity of $98 \%$ when compared with $\mathrm{CT}^{6}$

The management of SSCDS can range from guidance and reassurance in patients with mild symptoms to surgical treatment in severely affected patients with disabling symptoms. There is no drug treatment for SDCSS. For patients with symptoms induced by sound, avoidance of loud noises can be enough to prevent clinical manifestations. A ventilation tube can be beneficial for patients with symptoms resulting mainly from pressure in the external auditory canal. The superior semicircular canal can be approached through the mastoid or middle cranial fossa with obliteration (plugging) or repair (resurfacing) of the canal. In a series of 20 patients who underwent surgical correction of the middle fossa, the results were excellent, with complete resolution of symptoms in 15 (75\%) patients. ${ }^{4}$ In cases of significant autophonia, obliteration (plugging) improved symptoms in $94 \%$ of patients. ${ }^{13}$

The dehiscence involved in surgical intervention of the primary middle fossa is not associated with sensorineural hearing loss, and in some cases, it can lead to normalization of conductive hearing loss. ${ }^{14}$ Obliteration (plugging) achieves long-term control more often than repair (resurfacing). ${ }^{4}$ Revisional surgery to repair the middle fossa or stapes may be associated with stapes sensorineural hearing loss. ${ }^{14}$

\section{Conclusion}

SSCDS is considered a rare and treatable cause of vertigo, although its pathophysiologic aspects have not yet been fully elucidated. It should be included in the differential diagnosis of vertigo and hearing loss, particularly when acoustic reflexes are preserved.

As in the case reported here, in which vestibular symptoms were absent, a minority of patients have exclusively auditory manifestations. Signs and symptoms of the syndrome are rarely obvious, and a minimum workup is required to rule out or make diagnosis more probable and thus avoid misconduct.

\section{References}

1 Minor LB, Solomon D, Zinreich JS, Zee DS. Sound- and/or pressure-induced vertigo due to bone dehiscence of the superior semicircular canal. Arch Otolaryngol Head Neck Surg $1998 ; 124: 249-258$

2 Carey JP, Minor LB, Nager GT. Dehiscence or thinning of bone overlying the superior semicircular canal in a temporal bone survey. Arch Otolaryngol Head Neck Surg 2000;126:137-147

3 Ferreira SC, Lima MAMT. Superior canal dehiscence syndrome. Rev Bras Otorrinolaringol (Engl Ed) 2006;72:414-418

4 Minor LB. Clinical manifestations of superior semicircular canal dehiscence. Laryngoscope 2005;115:1717-1727

5 Minor LB, Cremer PD, Carey JP, Della Santina CC, Streubel SO, Weg N. Symptoms and signs in superior canal dehiscence syndrome. Ann N Y Acad Sci 2001;942:259-273

6 Krombach GA, Schmitz-Rode T, Haage P, et al. Semicircular canal dehiscence: comparison of T2-weighted turbo spin-echo MRI and CT. Neuroradiology 2004;46:326-331

7 Nam EC, Lewis R, Nakajima HH, Merchant SN, Levine RA. Head rotation evoked tinnitus due to superior semicircular canal dehiscence. J Laryngol Otol 2010;124:333-335

8 Rosowski JJ, Songer JE, Nakajima HH, Brinsko KM, Merchant SN. Clinical, experimental, and theoretical investigations of the effect of superior semicircular canal dehiscence on hearing mechanisms. Otol Neurotol 2004;25:323-332

9 Halmagyi GM, Aw ST, McGarvie LA, et al. Superior semicircular canal dehiscence simulating otosclerosis. J Laryngol Otol 2003; 117:553-557

10 Yuen HW, Boeddinghaus R, Eikelboom RH, Atlas MD. 15th Yahya Cohen Memorial Lecture: the relationship between the air-bone gap and the size of superior semicircular canal dehiscence. Ann Acad Med Singapore 2011;40:59-64

11 Belden CJ, Weg N, Minor LB, Zinreich SJ. CT evaluation of bone dehiscence of the superior semicircular canal as a cause of sound- and/or pressure-induced vertigo. Radiology 2003;226: 337-343

12 Cloutier JF, Bélair M, Saliba I. Superior semicircular canal dehiscence: positive predictive value of high-resolution CT scanning. Eur Arch Otorhinolaryngol 2008;265:1455-1460

13 Crane BT, Lin FR, Minor LB, Carey JP. Improvement in autophony symptoms after superior canal dehiscence repair. Otol Neurotol 2010;31:140-146

14 Limb CJ, Carey JP, Srireddy S, Minor LB. Auditory function in patients with surgically treated superior semicircular canal dehiscence. Otol Neurotol 2006;27:969-980 